\title{
Micro-nano fabrication of self-aligned silicon electron field emitter arrays using pulsed KrF excimer laser irradiation.
}

\begin{abstract}
Self-aligned silicon micro-nano structured electron field emitter arrays were fabricated using pulsed krypton fluoride (KrF) excimer laser crystallization (ELC) of hydrogenated amorphous thin silicon films (a-Si:H) on metal coated backplane samples using well established manufacturing techniques. We investigate the effect of laser beam profile, laser pulse frequency and sample stage scanning speed on the growth of micro-nano conical structures on the surface of the thin silicon films. Randomly oriented conical structures as high as $1 \mu \mathrm{m}$ were fabricated using laser scan frequency of $100 \mathrm{~Hz}$ and sample stage scanning speed of $0.25 \mathrm{~mm} / \mathrm{sec}$. Best field emission (FE) results were measured from samples with the highest surface features. FE currents in the order of $10^{-6} \mathrm{~A}$ and low turn-on emission threshold of $\sim 14 \mathrm{~V} / \mu \mathrm{m}$. Light emission from the prototype demonstrators was tested using bespoke driver electronics and planar anodes coated with indium tin-oxide (ITO) and low voltage FE phosphors, to exemplify their usage as cold-cathode emitters for future flat panel display technologies.
\end{abstract}

\section{Keywords:}

\section{INTRODUCTION}

Flat panel display technology (FPD) is a broad and rapidly evolving area. Conventional cathode-ray tube (CRT) displays had a dominant position in products such as television and computer monitors for many decades. But in recent years, FPDs have overtaken the CRT market share. The size of the FPD market is huge, estimated to be around $\$ 117$ billion by 2020 and is rapidly expanding [1]. Diverse display technologies are involved e.g. liquid crystal display (LCD) [2], organic light emitting diode display (OLED) [3], quantum dot light emitting diode display [4], etc and major technological and product breakthroughs are occurring on a regular basis. Field emission display (FED) is one such technology that is being extensively researched into finding the optimum electron emitter source that bombards a phosphor coating which acts as a light emission medium. Unlike CRT's, FED use a large array of fine metal tips or carbon nanotubes (CNT) positioned behind a phosphor coated anode plate to emit electrons through a process called electron emission [5]. FED's are considered to provide the closest matching picture quality to CRT's [6]. They are also energy efficient by consuming less power than existing LCD's and plasma displays. The major drawback that is preventing commercialization of this technology is the extensive photolithography required for micro-nano tip fabrication or development of new manufacturing facilities for large scale CNT deposition which increases the production cost.

Here, planar field emitters pave the way for moving this technology forward by using non-photolithography based fabrication of cold cathode field emitters [7-10]. Silicon being the backbone of the semiconductor industry, silicon production is an established manufacturing process. Hence the use of silicon as cold cathode field emitters leads towards the possibility of making commercially viable FED's [9]. Preliminary research in using pulsed excimer laser annealed planar silicon cold cathode emitters has shown that silicon has the potential to become the desired material for FED's [11-16]. Pulsed excimer laser crystallization (ELC) is also well known in the manufacturing world to produce crystalline silicon for use in electronics and active-matrix flat panel displays and other sensor technologies in the industry [17-19]. The key physical parameters of importance in selecting a suitable cold-cathode emitting material for FED application are, in addition to its longetivity, robustness and ability to readily integrate 
into an existing manufacturing process, the requirement to be able to source high current densities at relatively low electric fields. In addition, an ability to produce uniform electron emission current voltage characteristic with very little or no hysteresis is also essential, as the closeness of the electron emission curves with the applied field is important to design matrix driver strategies with the required precision, where suitable offset voltages can be used for switching gated cathodes.

\section{EXPERIMENTAL DETAILS}

Commercial grade undoped hydrogenated amorphous silicon (a-Si:H) thin films of thickness $100 \mathrm{~nm}$ were deposited by plasma enhanced chemical vapour deposition (PECVD) on $100 \mathrm{~nm}$ Molybdenum (Mo) coated Corning 7059 glass substrates. Deposition was carried out at substrate temperature of $250{ }^{\circ} \mathrm{C}$, RF power of $6.5 \mathrm{~W}$ and deposition pressure of $150 \mathrm{mtorr} . \mathrm{H}_{2} / \mathrm{SiH}_{4}+\mathrm{H}_{2}$ ratio was fixed at $20 \%$ and the gas flow rate to $100 \mathrm{sccm}$. Hydrogen content in the as-deposited samples was measured at $\sim 10 \%$. The films have an optical Tauc gap of $1.74 \mathrm{eV}$. The "gap" state defect density is expected to be below $10^{16} \mathrm{~cm}^{-3} \mathrm{eV}^{-1}$ in the middle of the gap. The films are slightly $n$ type due to structural (bonding) defect present in the a-Si:H. The samples were not dehydrogenated or pre-baked prior to ELC. The LPXpro 210 krypton fluoride (KrF) excimer laser ( $248 \mathrm{~nm}, 20 \mathrm{~ns}$ ) from Coherent GmbH (Germany) was used to irradiate the a-Si:H thin films, as illustrated in Fig. 1. The laser beam had to be homogenised to attenuate its spatial non-uniformities. A homogeniser and specially designed custom optics play the role of averaging out some of the spatial variations and to create a rectangular shaped "Top-Hat" symmetrical beam profile. The dimension of the rectangular shaped laser beam pattern is $4 \mathrm{~mm} \times 10 \mathrm{~mm}$ with sharp edges on all sides of the short $\mathrm{x}$-axis and the long y-axis with a top-flat beam profile (Fig. 2). As the sample is scanned across, the irradiated area on the a-Si:H thin films will see a sharp increase in laser fluence with the leading edge of the laser pulse, the laser fluence would then abruptly reduce to zero with the trailing edge. Multiple laser shots over each point of silicon are used to further minimise these in-homogenities as the laser is scanned over the film in steps smaller than the beam size. This process averages out the beam in-homogenities [19]. The deposited samples were excimer laser annealed in air and at room temperature using a "Top-Hat" beam profile at a surface melt-through laser fluence of $210 \mathrm{~mJ} / \mathrm{cm}^{2}$ and laser pulse frequency ranging from 20 to $100 \mathrm{~Hz}$. The laser annealing process was performed by mounting the samples on a motorized and computer-controlled translation stage from Aerotech (USA) at a variable scan rate ranging from 2 $\mathrm{mm} / \mathrm{sec}$ to $0.25 \mathrm{~mm} / \mathrm{sec}$ and scanned along the short X-axis as illustrated in Fig. 2. When the samples are scanned along the $\mathrm{x}$-axis relative to the laser beam, the a-Si:H thin films will be subjected to a sharp increase in laser fluence which quickly reaches its maximum and then abruptly reduces to zero. Scanning the samples along the long y-axis relative to the laser beam resulted in ablation of most of the top silicon and underlying metal layer, that can be attributed to the relatively long exposure time of the sample in the high laser fluence top-flat region of the laser beam. As described in the following sections, significant changes in surface morphology and FE characteristics were observed by varying the laser pulse frequency and sample stage scan rate.

Initial diode configuration FE measurements were conducted using a stainless steel spherical anode probe $(\sim 5 \mathrm{~mm}$ in diameter). The spherical anode probe is mounted on a piezo-ceramic actuator and controlled using a Burleigh 6000 micrometer allowing movement of the probe in sub-micron steps to set the required anode-cathode gap. The anode probe is positioned above the sample (cathode) at the required height from the cathode surface and a potential is applied between the anode probe and the cathode sample. This method is proven for diode FE measurements [20]. The applied electric field is increased every $30 \mathrm{sec}$ using a Keithley-248 high voltage power supply and the resulting FE current measurements were recorded using a Keithley-236 high resolution electrometer. The macroscopic electric field $\mathrm{E}_{\mathrm{TH}}(\mathrm{V} / \mu \mathrm{m})$ between the anode probe and silicon cathode is obtained by dividing the supplied anode voltage by the anode-cathode gap distance. During the FE tests, the anode voltage was ramped up and down for several cycles and the emission current versus measured electric field was recorded as the I-E curves. From the measured I-E curves we can obtain the FE threshold $\mathrm{E}_{\mathrm{TH}}(\mathrm{V} / \mu \mathrm{m})$. In our experiments the turn-on $\mathrm{E}_{\mathrm{TH}}$ is defined as the applied macroscopic field that produces a steady emission current not less than $1 \mathrm{nA}$. As a measure of the 
"hysteresis effect", the difference in the applied electric fields between the upward and downward voltage cycles at a current of $1 \mathrm{nA}$ is used. To visualize electron field emission, diode structured FED prototypes have been fabricated and tested. FE tests were carried out in a vacuum better than $4 \times 10^{-6}$ mbar.

\section{RESULTS \& DISCUSSION}

Surface profiling of the laser processed thin silicon films was performed using JEOL 6310 for scanning electron microscopy (SEM). Excimer laser processing of thin silicon films allows for uniform distribution of heat over the exposed area of the thin film, thus maintaining uniformity in the crystallization process. During laser processing, the samples are exposed to temperatures near to the ablation limit, this causes melting of the thin silicon film. The initial laser pulse would melt/quench silicon and dehydrogenate the film, thereby changing its absorption co-efficient. This cycle continues with the following pulses at higher laser frequencies, thereby creating a unique structure on the surface of the resultant material giving rise to the micro-nano scale conical structures. The first batch of thin silicon film samples were excimer laser annealed with a variable laser pulse frequency ranging from 20 to $100 \mathrm{~Hz}$ and fixed $\mathrm{X}-\mathrm{Y}$ stage sample scan rate of $2 \mathrm{~mm} / \mathrm{sec}$. Through this experiment, we observed that conical micro-nano structures on the surface of the laser processed a-Si:H thin films gradually evolved with the increasing laser frequency. The size and shape of the silicon micro-nano scale features on the surface were greatly influenced by the laser pulse frequency. Fig. 3, shows four SEM images taken from a-Si:H thin films irradiated with different laser pulse frequency's and fixed sample scan rate. At frequencies upto $20 \mathrm{~Hz}$, the a-Si:H thin films are fully crystallized and some nano and sub-micrometer scale protrusions can be seen on the surface of the film. Increasing the laser pulse frequency further to $40 \mathrm{~Hz}$, the solidified protrusions on the surface of the samples gradually grew in height and also became sharper at the top. The highest aspect ratio conical features with heights upto $1 \mu \mathrm{m}$ and twisted shape were measured at a laser pulse frequency of $100 \mathrm{~Hz}$. Any further increase in laser pulse frequency would result in gradual ablation of these conical structures. On examining the changes in the surface morphology of the a-Si:H thin films with different laser pulse frequency's, we are certain that micro-nano structuring of the a-Si:H thin films was due to the combination of the laser beam profile, surface melt through laser fluence and laser pulse frequency.

The second batch of the a-Si:H thin films were irradiated with a fixed laser pulse frequency of $20 \mathrm{~Hz}$ and variable $\mathrm{X}$ Y stage sample scan rate, micro-nano scale sharp conical structures could only be produced when the sample was scanned with a low sample stage scan rate of $0.25 \mathrm{~mm} / \mathrm{sec}$. Similar to our earlier experiment with variable laser pulse frequency, the size and shape of the micro-nano scale conical structures on the surface of the a-Si:H thin films was also influenced by the sample stage scan rate. Fig. 4, shows four SEM images of a-Si:H thin films irradiated at different sample scan rates and fixed laser pulse frequency of $20 \mathrm{~Hz}$. When the sample was laser processed at a sample scan rate of $2 \mathrm{~mm} / \mathrm{sec}$, the laser processed a-Si:H thin films are completely crystallized and sub-micrometer scale protrusions can be seen on the surface of the film. Reducing the sample scan rate to $1 \mathrm{~mm} / \mathrm{sec}$, the protrusions gradually grow in height and start becoming conical in shape with sharp top ends. The highest aspect ratio conical structures as high as $1 \mu \mathrm{m}$ and random orientation were fabricated at a sample scan rate of $0.25 \mathrm{~mm} / \mathrm{sec}$. Any further reduction in sample scan rate would result in the gradual ablation of these conical structures. It is believed that the traverse speed of the $\mathrm{X}-\mathrm{Y}$ stage modified the frequency of the laser beam as seen by the samples, resulting in a similar surface morphology as the samples that were processed with variable laser pulse frequency (Fig. 3). Hence by controlling the laser-beam profile, sample scan rate and laser pulse frequency we are able to control the shape and size of the randomly oriented, self-aligned, micro-nano scale structures on the surface of the metal backplane coated a-Si:H thin films.

Micro-nano engineering of conical silicon structures on silicon wafers using nano and femtosecond pulsed laser have been reported in some previous studies [21, 22]. We believe that a similar mechanism of conical structure formation can also be applied here to a-Si:H thin films. The initial pulses of the laser irradiation on the surface of the a-Si:H thin films results in the crystallization of the entire thin film. High laser fluence of the laser pulses would 
result in melting of the film and cause light ablation of the top layer of the thin film. The ablated material would redeposit on the surface and form impurity-rich cocoon-like protrusions that would shield the underlying material from erosion. These micro-nano scale conical silicon protrusions are beneficial for FE applications as they allow for low turn on electric field due to electrical field enhancement achieved from the sharp conical tips.

Due to manufacturing problems faced by earlier FE devices, CNTs were considered as the ideal cold cathode emitters, due to very high aspect ratio and mechanical stability at high electric fields. But concerns such as stray dominant emitters and proximity effects made them difficult to control and mass produce due to very low yield. Promising $\mathrm{E}_{\mathrm{TH}}$ have also been observed from amorphous carbon [9], diamond like carbon [10], planar amorphous silicon [9] and microcrystalline silicon [11]. Filamentary conduction in thin silicon films has reported stable FE currents after a break-down within the film [23-26]. This process is known as "conditioning", which results in the formation of stable conducting filaments within the thin films, thus allowing for high emission current densities and quantized resistance. Silva et al. has demonstrated that conditioning of thin silicon films using current-stressing resulted in structural changes in the films, thus allowing for very low $\mathrm{E}_{\text {Тн }}[9]$.

Field emission measurements indicate that ELC of a-Si:H thin films having sharp micro-nano scale conical structures on the surface exhibited the best electron field emission performance in terms of having the lowest $\mathrm{E}_{\mathrm{TH}}$ and largest measured emission currents. The representative field emission measurements of the laser irradiated aSi:H thin film shown in Fig. $3 \mathrm{a}$ and $3 \mathrm{~d}$ are presented in Fig. 5 and 6 respectively. This experiment allowed us to study the effect of high surface features on the FE characteristics. I-E curves shown in Fig. 5 and 6 were obtained with an anode cathode gap distance of $50 \mu \mathrm{m}$ and supplied anode voltage upto $3200 \mathrm{~V}$. By examining the I-E curve in Fig. 6, we observed that the $\mathrm{E}_{\mathrm{TH}}$ for $1 \mathrm{nA}$ electron current was less than $14 \mathrm{~V} / \mu \mathrm{m}$ for thin films with micrometer size conical structures (Fig. 3d) and $\mathrm{E}_{\mathrm{TH}}$ of $\sim 44 \mathrm{~V} / \mu \mathrm{m}$ (Fig. 5) for thin films with nano-scale crystalline cocoons on the surface (Fig. 3a). In both the FE measurements, the saturation current was measured upto several hundreds of micro-amperes. The data depicted in the both the I-E curves in Fig. 5 and 6 was also plotted as $\mathrm{Ln}\left(\mathrm{I} / \mathrm{E}^{2}\right)$ versus 1/E. The linear dependence of the Fowler-Nordheim plots in both the cases indicates that laser irradiated a-Si:H thin films indeed emit electrons through a field emission mechanism. In comparison to films with high conical features as shown in Fig. 3d, the films with nano scale protrusions on the surface (Fig. 3a) were measured to have poor FE performance. In most of the cases, stable and reproducible field emission currents could only be obtained after a high field conditioning cycle [9]. In our tests, the required conditioning cycle electric field was as high as $56 \mathrm{~V} / \mu \mathrm{m}$ (Fig. 5). Even after this high field conditioning process, the resultant $\mathrm{E}_{\mathrm{TH}}$ was still more than $40 \mathrm{~V} / \mu \mathrm{m}$. No such conditioning cycle was observed in the I-V curve of a-Si:H thin films with micro scale conical structures (Fig. 6). This can be attributed to field enhancement from the micro-nano conical structures. In addition, there is no evidence of significant hysteresis behavior between the upward and downward cycle of either I-E curves.

To visualize light emission from the excimer laser processed a-Si:H thin film cathodes, diode configured FED prototypes were fabricated and tested. For a matrix addressed diode structured FED device, the a-Si:H thin films were patterned on a Mo coated cathode plate and medium voltage FE green phosphor coated indium tin oxide (ITO) strips were patterned on the anode plate. A polymer spacer of $650 \mu \mathrm{m}$ thickness was sandwiched between the anode and cathode plates to maintain anode-cathode gap. Light emission pixels are formed at the intersection of the addressed anode and cathode. The video image in Fig. 7 was captured with the anode voltage of $9750 \mathrm{~V}$ and anodecathode separation of $650 \mu \mathrm{m}$, i.e., an electric field within the FED device is $15 \mathrm{~V} / \mu \mathrm{m}$. It was not possible to image individual emission sites, however the site density coverage was more than $80 \%$ at the applied electric field of 15 $\mathrm{V} / \mu \mathrm{m}$. Light emission tests were also performed in a vacuum better than $4 \times 10^{-6} \mathrm{mbar}$. FE at an applied field of 15 $\mathrm{V} / \mu \mathrm{m}$ compares favorably with that of emission from Si nanowires [27], better than FE from oxidation sharpened poly-Si [28], poly-Si microtips [29] and from single crystalline silicon micro-tips [30] at $\sim 20 \mathrm{~V} / \mu \mathrm{m}$.

In this paper, we have demonstrated that the growth of micro-nano scale conical structures and electron field emission characteristic from pulsed excimer laser processed a-Si:H thin silicon films not only strongly depends on the laser beam profile, but also the laser pulse frequency and the speed at which the samples are scanned. 
Homogeneous, high density micro-nano scale conical silicon structures can be fabricated on the surface of a-Si:H thin film by pulsed excimer laser irradiation with a symmetrical "Top-Hat" beam profile, surface melt-through laser fluence and by controlling the laser pulse frequency or the sample scan rate. Thin silicon film samples processed under these conditions possess good electron field emission properties with a low turn-on $\mathrm{E}_{\mathrm{TH}}$ of less than $14 \mathrm{~V} / \mu \mathrm{m}$ and a large saturated emission current of upto $740 \mu \mathrm{A} / \mathrm{cm}^{2}$. From these tests it has been exemplified that pulsed excimer laser processed a-Si:H thin films with micro-nano scale conical surface features are feasible for use as cold cathode emitters in large scale FED devices.

From the FE results discussed in the earlier section, it is believed that the micro-nano scale conical structures play a vital role in exhibiting electron field emission at low turn on $\mathrm{E}_{\mathrm{TH}}$ due to large field enhancement at the tips. However, on examining the conical Si structures, it can be seen that the aspect ratio of these structures is not high enough to initiate such strong field enhancement that would account for the measured low turn-on $\mathrm{E}_{\mathrm{TH}}$. It can be speculated that on irradiating the a-Si:H thin films with high laser fluence and sharp-edge "Top-Hat" beam profile, nano-sized quantum conducting filaments are formed that are buried within the film that could form high density electron emission sites and provide the necessary internal field enhancement within the bulk of the material. Experimentally, it has been observed that a-Si:H thin films annealed using the laser parameters discussed in this paper, contain a higher percentage of hydrogen. Typically, laser irradiation of a-Si:H thin films is equivalent to low temperature annealing and dehydrogenation process, thereby substantially releasing hydrogen from the samples. This would result in very high density of silicon dangling bond defects that can trap electrons and significantly decrease electron mobility within the material. When a-Si:H thin films are irradiate with a sharp edge "Top-Hat" beam profile, the quick melt through and re-solidification of the a-Si:H thin films would prevent the slow dehydrogenation process thereby preserving the hydrogen atoms within the films.

\section{CONCLUSION}

FED technology has been plagued by several manufacturing issues related to the fabrication of electron emitters. It is still a promising technology for future flat panel displays as it promises the technological advantage of an emissive display which currently OLED enjoys but without all the associated degradation and burn-in issues. The use of existing manufacturing processes for fabricating micro-nano electron emitting structures on thin silicon films using pulsed ELC paves the way for ease of adoption of this technology by the display industry, as it would allow for mass production of such displays at low cost. Excimer laser processing is central to the fabrication of the micronano emitters. By controlling the beam profile, laser pulse frequency and sample scan rate, we are able to fabricate self-aligned micro-nano silicon electron emitters of the desired shape and size. The underlying mechanism of FE from this material is still not fully understood but it is believed that field enhancement from the micro-nano emitting tips is also responsible for the FE process. However, encouraging FE and light emission tests from these micro-nano silicon structures, exemplifies the potential of this technology for future flat panel displays. 


\section{REFERENCES}

[1] Flat Panel Display Revenues Forecast to Increase in 2017, HIS Markit Says. Available online: https://technology.ihs.com/585847/flat-panel-display-revenues-forecast-to-increase-in-2017-ihs-markitsays (accessed on 22 October 2018).

[2] A.R. Kmetz, Liquid-crystal display prospects in perspective. Electron Devices, IEEE Trans. on, 20, 954 961, (1973)

[3] H.W. Chen, J. H. Lee, B.Y. Lin, S. Chen, S. T. Wu, Liquid crystal display and organic light-emitting diode display: present status and future perspectives. Light: Science \& Applications, 7, 17168, (2018)

[4] M. K. Choi, J. Yang, T. Hyeon, D. H. Kim, Flexible quantum dot light-emitting diodes for next generation displays. npj Flexible Electronics, 2, 10, (2018)

[5] B. F. Coll, K. A. Dean, E. Howard, S. V. Johnson, M. R. Johnson, J. E. Jaskie, Nano-emissive display technology for large-area HDTV. Journal of the Society for Information Display, 14, 477-485, (2006)

[6] C. A. Spindt, A thin film field emission cathode. J. Appl. Phys., 39, 3504, (1968)

[7] R. H. Fowler, L. Nordheim, Electron emission in intense electric fields. Proc. R. Soc. Lon. A, 119, (1928)

[8] L. W. Nordheim, The effect of the image force on the emission and reflexion of electrons by metals. Proc. R. Soc. Lon. A, 121, (1928)

[9] S.R.P. Silva, R. D. Forrest, J. M. Shannon, Electron field emission from amorphous silicon. J. Vac. Sci. Tech. B, 17, 596, (1999)

[10] G. Amaratunga, S.R.P. Silva, Nitrogen containing hydrogenated amorphous carbon for thin-film field emission cathodes. Appl. Phys. Lett., 68, 2529, (2002)

[11] Y. F. Tang, S.R.P. Silva, J. Shannon, M. J. Rose, Electron field emission from excimer laser crystallized amorphous silicon. Appl. Phys. Lett., 80, 4154, (2002)

[12] M. J. Rose, C. Main, Y. Fan, S. K. Persheyev, M. Z. Shaikh, S. Silva, Laser processed hydrogenated amorphous silicon for field emission displays, J. Optoelectron. Adv. M., 11, 1037-1043, (2009)

[13] M. Z. Shamim, S. K. Persheyev, M. J. Rose, Electron field emission display based on AIC-PECVD thin silicon films, IEEE Proc. 10th Int. Conf. on Intelligent Sys. \& Control, Coimbatore, India, 7-8 Jan. (2016)

[14] M. Z. Shaikh, K. A. O’Neill, S. K. Persheyev, M. J. Rose, Excimer Laser Crystallized HWCVD Thin Silicon Films: Electron Field Emission, MRS Proceedings, USA, (2005)

[15] K. A. O'Neill, M. Z. Shaikh, G. Lyttle, S. Anthony, Y. C. Fan, S. K. Persheyev, M. J. Rose, Laser annealed HWCVD and PECVD thin silicon films. Electron field emission. Thin Solid Films, 501, 310-31, (2006)

[16] M. Z. Shaikh, K. A. O’Neill, S. Anthony, S. K. Persheyev, M. J. Rose, Optical and electronic properties of HWCVD and PECVD silicon films irradiated using excimer and Nd:Yag lasers. Thin Solid Films, 501, $125-128,(2006)$ 
[17] K. Shimizu, O. Sugiura, M. Matsumura, On-Chip Bottom-Gate Polysilicon and Amorphous Silicon ThinFilm Transistors Using Excimer Laser Annealing. Jpn. J. Appl. Phys., 29, L1775, (1990)

[18] I. Asai, N. Kato, M. Fuse, Poly-Silicon Thin-Film Transistors with Uniform Performance Fabricated by Excimer Laser Annealing. Jpn. J. Appl. Phys., 32, 474, (1993)

[19] H. Kuriyama, T. Kuwahara, S. Ishida, T. Nohda, K. Sano, H. Iwata, S. Noguchi, S. Kiyama, S. Tsuda, S. Nakano, Improving the Uniformity of Poly-Si Films Using a New Excimer Laser Annealing Method for Giant-Microelectronics. Jpn. J. Appl. Phys., 31, 4550, (1992)

[20] R. D. Forrest, A. P. Burden, S. R. P. Silva, L. K., Cheah, A study of electron field emission as a function of film thickness from amorphous carbon films, Appl. Phys. Lett., 73, 3784, (1998)

[21] V. Zorba, I. Alexandrou, I. Zergioti, A. Manousaki, C. Ducati, A. Neumeister, C. Fotakis, G.A.J.Amaratunga, "Laser microstructuring of Si surface for low-threshold field-electron emission", Thin Solid Films, 453, pp.492-495, (2004)

[22] B. R. Tull, J. E. Carey, E. Mazur, J. McDonald and S. M. Yalisove, "Surface morphologies of silicon surfaces after femtosecond laser irradiation", Mat. Res. Soc. Bull., 31, pp.626-633, (2006)

[23] J. Hajto, A. E. Owen, A. J. Snell, P. G. Le Comber, M. J. Rose, Analogue memory and ballistic electron effects in metal-amorphous silicon structures, Philosophical Magazine B, 63:1, 349-369, (1991)

[24] A. E. Owen, J. Hajto, A. J. Snell, P. G. Le Comber, M. J. Rose, Analogue memory effects in metal/aSi:H/Metal memory devices, J. Non Cryst. Sol, 137-138, 1257-1262, (1991)

[25] J. Hajto, A. E. Owen, A. J. Snell, P. G. Le Comber, M. J. Rose, S. M. Gage, Quantized electron transport in amorphous-silicon memory structures, Phys. Rev. Lett., 66, 1918, (1991)

[26] J. Hu, J. Hajto, A. J. Snell, M. J. Rose, Electron transport in metal-amorphous silicon-metal memory devices, IEICE Transactions on Electronics, E84C(9), 1197,(2001)

[27] F. C. K. Au, K. W. Wong, Y. H. Tang, Y. F. Zhang, I. Bello, and S. T. Lee, Electron field emission from silicon nanowires, Appl. Phys. Lett. 75, 1700, (1999).

[28] A. A. Evtukh, Influence of surface morphology of the polycrystalline silicon on field electron emission, Thin Solid Films 337, 261 (1999).

[29] S. E. Huq, G. H. Grayer, and P. D. Prewett, Comparative study of gated single crystal silicon and polysilicon field emitters, J. Vac. Sci. Technol. B 15, 2855 (1997).

[30] T. Sugino, S. Kawasaki, K. Tanioka, and J. Shirafuji, Electron emission from boron nitride coated Si field emitters, Appl. Phys. Lett. 71, 2704 (1997). 


\section{FIGURE CAPTIONS}

Fig. 1. Schematic representation of excimer laser annealing process of thin silicon film mounted on a moving $\mathrm{X}-\mathrm{Y}$ stage.

Fig. 2. Top-Hat beam profile of the $248 \mathrm{~nm}, 4 \times 10 \mathrm{~mm}^{2}$ laser pulse. Dimensions measured at the sample plane.

Fig. 3. SEM micrograph of thin silicon films annealed at a fixed $X-Y$ stage sample scan speed of $2 \mathrm{~mm} / \mathrm{sec}$ and varying laser pulse frequency of a) $20 \mathrm{~Hz}$, b) $40 \mathrm{~Hz}$, c) $80 \mathrm{~Hz}$ and d) $100 \mathrm{~Hz}$.

Fig. 4. SEM micrograph of ELC thin silicon films annealed at a fixed laser pulse frequency of $20 \mathrm{~Hz}$ and varying X-Y stage sample scan speed of a) $2 \mathrm{~mm} / \mathrm{sec}$ (b) $1 \mathrm{~mm} / \mathrm{sec}$, (c) $0.5 \mathrm{~mm} / \mathrm{sec}$ and (d) $0.25 \mathrm{~mm} / \mathrm{sec}$.

Fig. 5. I-E curve of ELC thin silicon film with sub micrometer scale protrusions on the surface. $\mathrm{E}_{\mathrm{TH}}=\sim 44 \mathrm{~V} / \mu \mathrm{m}$ (Anode-Cathode gap $=50 \mu \mathrm{m})$

Fig. 6. I-E curve of ELC thin silicon film with micro-nanoscale conical structures on the surface. $\mathrm{E}_{\mathrm{TH}}=\sim 14 \mathrm{~V} / \mu \mathrm{m}$ $($ Anode-Cathode gap $=50 \mu \mathrm{m})$

Fig. 7. Green light emission from a FED prototype using excimer laser processed a-Si:H films as cold cathode emitters. Active area $=2 \mathrm{~cm} \times 1.5 \mathrm{~cm}$ 